\title{
Prevalence Estimates of ADHD in a Sample of Inpatients With Alcohol Dependence
}

\author{
Mathias Luderer ${ }^{1,2} \oplus$, Christian Sick ${ }^{4}$, Nurcihan Kaplan-Wickel ${ }^{4}$, \\ Iris Reinhard ${ }^{3}$, Agnes Richter ${ }^{4}$, Falk Kiefer ${ }^{1,2}$, and Tillmann Weber ${ }^{4}$
}

\begin{abstract}
Objective: ADHD is common in patients with alcohol dependence, but prevalence results are inconsistent. We investigated ADHD prevalence in a complex design to avoid over- or underdiagnosing. Method: Patients with alcohol dependence starting long-term residential treatment were included. A structured interview (Diagnostic Interview for ADHD in Adults [DIVA]) was conducted on all patients. DIVA results indicating childhood or adulthood ADHD were assessed in successive diagnostic interviews by two expert clinicians. Results: 415 of 488 patients had completed the entire diagnostic assessment. ADHD prevalence was $20.5 \%$. DIVA results correlated moderately with experts' diagnoses. In patients with ADHD, a higher comorbid illicit substance use was prevalent and alcohol dependence started earlier and was more severe. Conclusion: This study provides the largest sample on ADHD prevalence in alcohol dependent inpatients. Despite great efforts to avoid overestimation, we found every fifth patient to have ADHD. ADHD diagnosis should not be based solely on a structured interview but should be clinically confirmed. (. of Att. Dis. 2020;24(I 4) 2072-2083)
\end{abstract}

\section{Keywords}

dual disorder, structured interview, alcohol, substance related disorders

\section{Introduction}

\section{ADHD and Substance Use Disorders (SUDs)}

ADHD is common but often underrecognized (Huntley et al., 2012; McAweeney, Rogers, Huddleston, Moore, \& Gentile, 2010) in patients with SUD. Comorbid ADHD in SUD patients has a negative impact on SUD treatment efficacy and treatment retention (Arias et al., 2008; Carroll \& Rounsaville, 1993; Ercan, Coskunol, Varan, \& Toksoz, 2003; Wilens \& Morrison, 2011) and is associated with a more severe course of substance use, social, and psychiatric impairment (Moura et al., 2013).

ADHD during childhood or adolescence increases the risk for developing an alcohol use disorder (AUD) or other SUDs (Lee, Humphreys, Flory, Liu, \& Glass, 2011; Wilens $\&$ Morrison, 2011). Also, ADHD is a risk factor for heavy alcohol use and initiation of illicit drug use in young adults (Vogel et al., 2016). Some studies have suggested that this increased risk can be explained by shared genetics (Capusan, Bendtsen, Marteinsdottir, Kuja-Halkola, \& Larsson, 2015; Edwards \& Kendler, 2012), while psychological factors may also contribute to enhance the risk for substance use: (emotional) impulsivity (De Alwis, Lynskey, Reiersen, \& Agrawal, 2014; Pedersen et al., 2016; Roberts, Peters, Adams, Lynam, \& Milich, 2014) and aversion to delay gratification (Wickens \& Tripp, 2005) as well as neuroticism and anxiety (Davis, Cohen, Davids, \& Rabindranath, 2015) may mediate between ADHD symptoms and early inclination toward substance use.

ADHD has a worldwide prevalence in the general population of $3.4 \%$ in childhood and adolescence (Polanczyk, Salum, Sugaya, Caye, \& Rohde, 2015) and of $2.5 \%$ in adults (Simon, Czobor, Balint, Meszaros, \& Bitter, 2009). In the AUD population, prevalence is increased with reported prevalence rates ranging from $6.6 \%$ to $21.3 \%$ (Daigre et al., 2015; Johann, Bobbe, Putzhammer, \& Wodarz, 2003; Ohlmeier et al., 2008; Reyes et al., 2019; Roncero et al., 2019; van de Glind et al., 2014).

'Dept. of Addictive Behavior and Addiction Medicine, Central Institute of Mental Health (CIMH), Heidelberg University, Medical Faculty Mannheim, Germany

${ }^{2}$ Feuerlein Center of Translational Addiction Medicine

${ }^{3}$ Dept. of Biostatistics, Central Institute of Mental Health, Heidelberg

University, Medical Faculty Mannheim, Germany

${ }^{4}$ MEDIAN Klinik Wilhelmsheim, Oppenweiler, Germany

\section{Corresponding Author:}

Mathias Luderer, Department of Addictive Behavior and Addiction Medicine, Central Institute of Mental Health, Medical Faculty Mannheim, Heidelberg University, Square J5, Mannheim 68159, Germany.

Email: mathias.luderer@zi-mannheim.de 


\section{Diagnosing ADHD in Patients With SUD}

Structured interviews for the diagnostic assessment of ADHD in SUD populations are frequently used but have not yet been validated for this group (Ramos-Quiroga et al., 2019). However, withdrawal and intoxication symptoms or psychological consequences from chronic substance use (i.e., substance-induced disorders) might interfere with the diagnostic process. Furthermore, early abstinence in AUD is also associated with increased symptoms of depression and anxiety, sleep disturbances, increased sensitivity to stress, anhedonia, and emotional dysregulation (Heilig, Egli, Crabbe, \& Becker, 2010; Spanagel, Noori, \& Heilig, 2014). Furthermore, this period is associated with a hypodopaminergic state in humans and rodents and with increased motor activity in animal models (Hirth et al., 2016). Hence, observed symptoms might be wrongly attributed to SUD, other psychiatric disorders, or ADHD. Therefore, the longitudinal aspect of ADHD as a nonepisodic disorder and the association of ADHD symptoms with periods of abstinence or substance use have to be taken into account carefully (Levin \& Upadhyaya, 2007; Ramos-Quiroga et al., 2019; Sullivan \& Rudnik-Levin, 2001). Moreover, novelty of the therapeutic setting, sobriety by itself, and initiation of psychotherapy may initially aggravate affective instability and stress sensitivity. Thus, an extended period of stabilization during prolonged abstinence might be required to unequivocally assess ADHD symptoms in AUD patients.

\section{Aims of This Study}

We conducted a study on ADHD prevalence in alcohol dependent patients that takes sufficient account of the risk of under- and overdiagnosing ADHD: (a) instead of relying solely on a structured interview, ADHD diagnosis had to be confirmed clinically by two experts in the field; (b) the diagnostic process started several weeks after hospital admission to exclude interference with intoxication, (prolonged) withdrawal, or chronic substance use symptoms and to ensure emotional, mental, and social stabilization in a residential long-term treatment setting; (c) a great amount of additional information on childhood and current ADHD symptoms was gathered (school records, parent ratings, behavior during treatment, ratings of close friends/relatives); and (d) the residential long-term treatment setting resulted in low treatment discontinuation and study dropout rates which allowed to fully assess the majority of patients over the course of many weeks.

\section{Method}

At the addiction treatment center MEDIAN Klinik Wilhelmsheim, Germany, approximately 750 patients (age $>18$ years) with alcohol dependence are treated per year in a residential setting after elective admission, each treatment lasting 8 to 16 weeks. Relapse and early discharge are rare events and patients are required to be abstinent on admission.

\section{Study Design}

The study had no external funding and was approved by the local ethics committee. Main inclusion criteria were written informed consent and a diagnosis of alcohol dependence according to International Statistical Classification of Diseases and Related Health Problems-10 (ICD-10; World Health Organization, 1993). Exclusion criteria were serious cognitive deficits. The study design is illustrated in Figure 1. All patients received information on the study and ADHD in a group setting before informed consent was obtained. After 4 to 5 weeks of inpatient treatment, a structured interview on ADHD (Diagnostic Interview for ADHD in Adults-2.0 [DIVA-2.0]) was conducted by two medical doctors. DIVA is free of charge and available in many different languages including German (J. J. S. Kooij, 2012). DIVA uses the Diagnostic and Statistical Manual of Mental Disorders (4th ed.; DSM-IV; American Psychiatric Association [APA], 1994) ADHD criteria for childhood and adulthood and elucidates ADHD criteria with different examples. The interview takes 45 to $90 \mathrm{~min}$.

\section{Preventing Over- and Underdiagnosing of ADHD}

To ensure that administration of DIVA would not lead to overdiagnosis, two experts in the field of ADHD/SUD, each with several years of clinical expertise in psychiatry and addiction medicine and specialized in diagnosing and treating comorbid ADHD, had to clinically confirm each suspected ADHD diagnosis made by DIVA in successive nonstructured clinical interviews.

When diagnosing ADHD during adulthood, Diagnostic and Statistical Manual of Mental Disorders (5th ed.; DSM5; APA, 2013) requires five or more symptoms of inattention and/or hyperactivity-impulsivity and "several" symptoms before the age of 12 retrospectively. When diagnosing ADHD during childhood or adolescence, six or more symptoms are needed. Irrespective of the time of diagnosis, $D S M-I V$ requires six or more symptoms both for adults and children prior to 7 years of age. To ensure that administration of DIVA would not lead to underdiagnosis, we lowered the diagnostic threshold for suspicious DIVA below DSM-IV and $D S M-5$ criteria. In this study, only four or more symptoms in at least one cluster during adulthood or five or more symptoms in at least one cluster during childhood were sufficient to initiate further diagnostic assessment. Hence, persisting ADHD symptoms from child- to adulthood were not required for further diagnostic assessment. In a stepwise approach, Expert 1 would see all patients with suspicious 

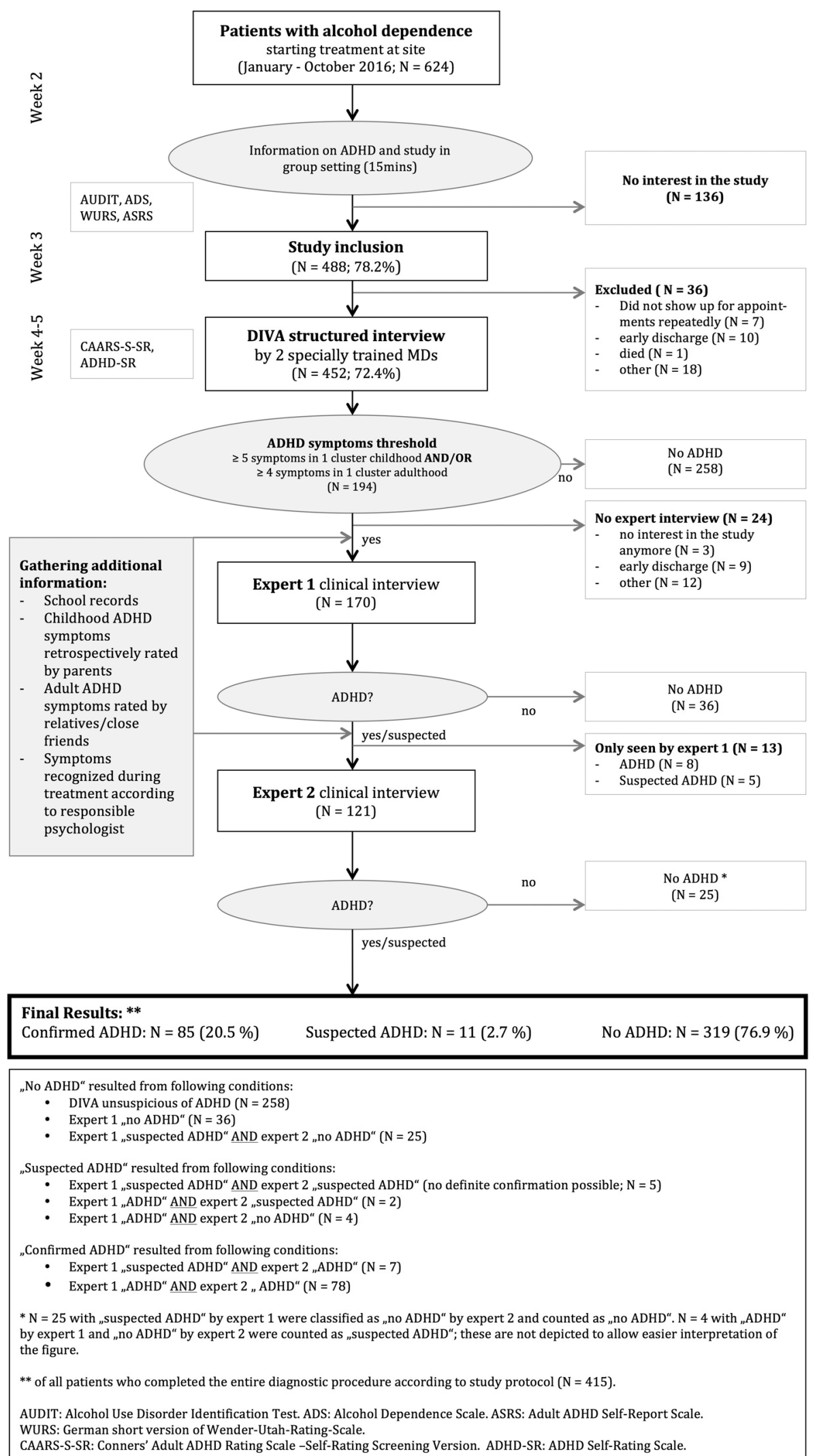

* $\mathrm{N}=25$ with "suspected ADHD“ by expert 1 were classified as „no ADHD“ by expert 2 and counted as "no ADHD“. $N=4$ with "ADHD“ by expert 1 and „no ADHD“ by expert 2 were counted as "suspected ADHD“; these are not depicted to allow easier interpretation of the figure.

** of all patients who completed the entire diagnostic procedure according to study protocol $(\mathrm{N}=415)$.

AUDIT: Alcohol Use Disorder Identification Test. ADS: Alcohol Dependence Scale. ASRS: Adult ADHD Self-Report Scale. WURS: German short version of Wender-Utah-Rating-Scale.

CAARS-S-SR: Conners' Adult ADHD Rating Scale -Self-Rating Screening Version. ADHD-SR: ADHD Self-Rating Scale.

Figure I. Study design and diagnostic process. 
Table I. Additional Information Resources for ADHD Symptoms.

Self-rating childhood ADHD symptoms

Self-rating adulthood ADHD symptoms

Informant rating childhood ADHD symptoms

Informant rating adulthood ADHD symptoms

Information from responsible psychologists during residential treatment
- German short version of the Wender Utah Rating Scale (Retz-Junginger et al., 2003; Ward, Wender, \& Reimherr, 1993)

- Conners' Adult ADHD Rating Scale-Self-Rating Screening Version (Conners, Erhardt, \& Sparrow, 1999)

- Adult ADHD Self-Report Scale vl.I (ASRS; Kessler et al., 2005)

- ADHD Self-Rating Scale (Rosler et al., 2004)

- Parent Rating Scale (Krause, Krause, \& Trott, 1998; Wender, 1995)

- School records for the age of 6 to 12 years

- Close friends/relatives: Conners' Adult ADHD Rating Scale-Informant Rating Screening Version (Conners et al., 1999)

- If ADHD had been diagnosed before, medical records were reviewed.

- Patient's performance and behavior (nonstructured)

- ADHD diagnostic checklist (Rosler et al., 2004)
DIVA results whereas Expert 2 would only see patients deemed suspicious of ADHD by Expert 1. If Expert 1 rejected ADHD diagnosis, Expert 2 would not additionally interview these patients.

The experts used a three-staged scoring system ("no ADHD," "suspected ADHD," "ADHD"). The diagnostic verdict of Expert 1 was available to Expert 2. If experts' opinions did not match, cases were discussed. If a unanimous decision could not be made, ADHD was not diagnosed (see Figure 1).

\section{Performance of the Structured Interview}

Experts' diagnoses were compared with DIVA for those patients who had suspicious DIVA results (with the lowered threshold mentioned above) and had received the required expert interviews according to protocol. This allows an estimation of DIVA's performance in AD patients. For this purpose, we correlated experts' diagnoses with DIVA results at different cutoffs for number of symptoms per cluster (hyperactivity/ impulsivity or inattention) in childhood and adulthood.

\section{Additional Sources of Information}

An extensive amount of additional information was gathered (Table 1). These resources were considered in the experts' clinical diagnostic assessment. The following collateral data were obtained: structured information from clinical staff $(n=149)$, informants' ratings adulthood $(n=$ $126)$, parents ratings of childhood $(n=91)$, and school records $(n=53)$.

\section{Other Variables Assessed}

Substance use and SUD diagnoses according to ICD-10 were routinely assessed at treatment initiation. Demographic data and other diagnoses were retrieved from patients' files.

Two questionnaires regarding alcohol addiction were completed during the second week after admission. The
Alcohol Dependence Scale (ADS) is a self-rating instrument to assess severity of alcohol dependence (Skinner \& Horn, 1984). The Alcohol Use Disorders Identification Test (AUDIT) is a widely used screening test to identify problematic alcohol consumption (Saunders, Aasland, Babor, de la Fuente, \& Grant, 1993).

Alcohol abstinence was verified by regular alcohol breath tests: Each day, a random selection of 40 patients (number of hospital beds: 214) was assessed. In addition, alcohol breath tests, urine ethyl glucuronide (EtG), and urine toxicology for illicit drugs, opioids, and benzodiazepines were conducted on a regular base in certain patients or upon request from staff when patients were suspected of substance intake.

\section{Statistical Analyses}

Demographic and clinical characteristics, severity of alcohol dependence (AUDIT and ADS), and DIVA results were compared between groups (ADHD vs. no ADHD) using chi-square or Fisher's exact test and Mann-Whitney $U$ tests. For comparison between DIVA and experts' diagnoses, Cohen's kappa was calculated for those patients who had suspicious DIVA results and completed diagnostic assessment per protocol. Values between 0.6 and 0.79 are considered moderate levels of agreement (McHugh, 2012). For all calculations, results with $p<.05$ were considered significant.

\section{Results}

\section{Participants and Inclusion Rates}

Study recruitment took place from January until October 2016. 488 of 624 admitted alcohol dependent patients (78.2\%) agreed to participate in the study. In 36 patients, DIVA could not be conducted and patients were thus excluded from the study (Figure 1). Hence, 452 patients with alcohol dependence ( $72.4 \%$ of all admitted patients) 
received a DIVA interview at 4 to 5 weeks after treatment initiation. 24 of 452 patients (4.6\%) had suspicious DIVA results but were not further seen by any expert (Figure 1). Another 13 patients (2.9\%) completed DIVA and one expert interview but the scheduled second expert interview was not conducted. In total, 415 of 488 patients $(85 \%)$ completed the entire study according to protocol with one to three interviews spread out over several weeks. Mean time between DIVA and final expert interview was $24.7 \pm 16.4$ days (median $=22.0 \pm 10.3$; range $=4-105$ ), resulting in an average period of approximately 8 weeks from admission to final diagnosis.

\section{Study Dropout}

24 patients had DIVA results indicating an expert interview (suspecting ADHD diagnosis) but no expert interview could be conducted. These patients who dropped out of the study directly after the DIVA interview showed significant differences in several variables (Supplementary Table 1) that resemble those of ADHD patients: They were significantly younger on admission, had a significantly younger age of onset of problematic alcohol use, had more drinking days on admission, had a higher relapse rate during treatment, and showed a higher frequency of any SUD (especially cannabis and polydrug use disorder).

Due to incomplete data, this analysis could not be executed for those patients who were excluded before DIVA.

\section{ADHD Prevalence}

In a strict per protocol analysis, only those study patients who had completed the entire diagnostic procedure $(n=$ $415)$ were included. 85 patients $(20.5 \%)$ were diagnosed with ADHD. Only five of these $85(5.9 \%)$ patients had been diagnosed with adult ADHD prior to hospital admission, two of them receiving medical treatment with methylphenidate or atomoxetine on admission. All adult ADHD diagnoses known on admission were confirmed in our study. No additional patients with a known diagnosis of childhood or adolescent ADHD were identified in our study population. In 11 patients (2.7\%), the diagnosis "suspected ADHD" resulted from either both experts not being able to confirm or reject ADHD $(n=5)$ or Expert 2 not being able to confirm the (suspected) ADHD diagnosis of Expert $1(n=6)$. Two patients were diagnosed with "late-onset" ADHD as they showed and reported typical ADHD symptoms and functional deficits in adulthood but had only few or no ADHD symptoms before the age of 12 .

\section{Performance of the Structured Interview}

DIVA results were correlated with final ADHD diagnosis. For this analysis, patients with experts' diagnoses of "suspected ADHD" $(n=11)$ as well as patients who did not complete the diagnostic procedure as per protocol were excluded $(n=37)$. Thus, 146 patients who were above our self-defined threshold for suspicious DIVA results received expert interviews according to protocol. This analysis does not include the 258 patients whose DIVA scores were below our self-defined threshold, as no further diagnostic assessment was performed.

Depending on the chosen threshold values (Table 2), DIVA showed only moderate agreement with the experts' opinions (Cohen's Kappa $=.514-.579)$. Agreement was best $($ Kappa $=.579$ and .563$)$ when DSM-5 criteria for adult ADHD (five or more symptoms in at least one cluster for adulthood) were applied together with four to five or more childhood symptoms in at least one cluster.

\section{Substance Use Variables and ADHD}

Substance use variables are shown in Table 3. The majority of patients with and without ADHD had been detoxified prior to hospital admission as required for residential rehabilitation treatment in Germany. Alcohol relapse during treatment occurred infrequently, but patients with ADHD relapsed twice as often $(10.6 \%$ vs. $4.4 \% ; p=.036)$. Patients with ADHD were significantly younger (41.4 vs. 49.3 years; $p<.001)$ on admission but had the same duration of alcohol dependence (13.1 vs. 13.5 years; $p=.681$ ). Hence, alcohol dependence started at a younger age in patients with ADHD, who also showed a more severe alcohol dependence both in the ADS (18.6 vs. $12.7 ; p<.001)$ and AUDIT ( 28.5 vs. $25.0 ; p<.001)$. They also reported a higher rate of previous delirium tremens $(14.1 \%$ vs. $6.0 \% ; p=.012)$, but not of withdrawal seizures ( $15.3 \%$ vs. $15.2 \% ; p=.611)$.

In patients with ADHD, tobacco use disorder started at an earlier age (16.2 vs. 18.1 years; $p=.010)$. The rate of tobacco dependence was increased but did not reach statistical significance $(80.0 \%$ vs. $69.3 \% ; p=.052)$. Patients with ADHD also had a higher rate of comorbid drug use disorder $(32.9 \%$ vs. $11.6 \% ; p<.001)$, in particular cannabis $(23.5 \%$ vs. $7.8 \% ; p<.001)$, and a higher rate of past intravenous drug abuse $(7.1 \%$ vs. $1.6 \% ; p=.014)$.

\section{Discussion}

This study provides the largest sample on ADHD prevalence in $\mathrm{AD}$ inpatients reported so far. The prevalence rate of $20.5 \%$ in the present study is among the highest in the literature (Daigre et al., 2015; Johann et al., 2003; Ohlmeier et al., 2008; Reyes et al., 2019; Roncero et al., 2019; van de Glind et al., 2014; van Emmerik-van Oortmerssen et al., 2012) despite eliminating many of the usual risks that might lead to overdiagnosing ADHD.

Recent studies on ADHD prevalence in AD patients reported a broad range of $6.6 \%$ to $21.3 \%$ (Daigre et al., 
Table 2. Results of Structured ADHD Interview (DIVA) in Comparison With Experts' Diagnoses With Different Cutoff Values for Childhood and Adulthood Symptoms.

\begin{tabular}{|c|c|c|c|c|c|c|c|c|c|}
\hline \multirow[b]{2}{*}{ DIVA cutoff values } & \multirow[b]{2}{*}{ DIVA result } & \multicolumn{2}{|c|}{ Experts' diagnoses } & \multirow{2}{*}{$\begin{array}{l}\text { Cohen's } \\
\text { Kappa }\end{array}$} & \multirow[b]{2}{*}{ Sens. } & \multirow[b]{2}{*}{ Spec. } & \multirow[b]{2}{*}{ PPV } & \multirow[b]{2}{*}{ NPV } & \multirow[b]{2}{*}{$p$} \\
\hline & & ADHD & No ADHD & & & & & & \\
\hline $\begin{array}{l}\text { Threshold for expert interview } \\
\text { Childhood } \geq 5 \text { symptoms in I cluster } \\
\text { and/or } \\
\text { Adulthood } \geq 4 \text { symptoms in I cluster }\end{array}$ & $\begin{array}{c}\text { DIVA above } \\
\text { threshold }\end{array}$ & 85 & 61 & - & - & - & - & - & $<.001 * *$ \\
\hline \multirow{2}{*}{$\begin{array}{l}\text { DSM-IV } \\
\text { Childhood } \geq 6 \text { symptoms in I cluster } \\
\text { and } \\
\text { Adulthood } \geq 6 \text { symptoms in I cluster }\end{array}$} & DIVA ADHD & 66 & 14 & .541 & .78 & .77 & .83 & .71 & $<.001 * *$ \\
\hline & $\begin{array}{c}\text { DIVA no } \\
\text { ADHD }\end{array}$ & 19 & 47 & & & & & & \\
\hline \multirow{2}{*}{$\begin{array}{l}\text { DSM-5: Childhood } 3 \\
\text { Childhood } \geq 3 \text { symptoms in I cluster } \\
\text { and } \\
\text { Adulthood } \geq 5 \text { symptoms in I cluster }\end{array}$} & DIVA ADHD & 78 & 26 & .514 & .92 & .57 & .75 & .83 & $<.001 * *$ \\
\hline & $\begin{array}{c}\text { DIVA no } \\
\text { ADHD }\end{array}$ & 7 & 35 & & & & & & \\
\hline \multirow{2}{*}{$\begin{array}{l}\text { DSM-5: Childhood } 4 \\
\text { Childhood } \geq 4 \text { symptoms in I cluster } \\
\text { and } \\
\text { Adulthood } \geq 5 \text { symptoms in I cluster }\end{array}$} & DIVA ADHD & 77 & 22 & .563 & .91 & .64 & .78 & .83 & $<.001 * *$ \\
\hline & $\begin{array}{c}\text { DIVA no } \\
\text { ADHD }\end{array}$ & 8 & 39 & & & & & & \\
\hline \multirow{2}{*}{$\begin{array}{l}\text { DSM-5: Childhood } 5 \\
\text { Childhood } \geq 5 \text { symptoms in I cluster } \\
\text { and } \\
\text { Adulthood } \geq 5 \text { symptoms in I cluster }\end{array}$} & DIVA ADHD & 77 & 21 & .579 & .91 & .66 & .79 & .83 & $<.001^{* *}$ \\
\hline & $\begin{array}{r}\text { DIVA no } \\
\text { ADHD }\end{array}$ & 8 & 40 & & & & & & \\
\hline \multirow{2}{*}{$\begin{array}{l}\text { DSM-5: Childhood } 6 \\
\text { Childhood } \geq 6 \text { symptoms in I cluster } \\
\text { and } \\
\text { Adulthood } \geq 5 \text { symptoms in I cluster }\end{array}$} & DIVA ADHD & 71 & 18 & .545 & .84 & .70 & .80 & .75 & $<.001 * *$ \\
\hline & $\begin{array}{c}\text { DIVA no } \\
\text { ADHD }\end{array}$ & 14 & 43 & & & & & & \\
\hline
\end{tabular}

Note. Patients with "suspected ADHD" $(n=\mathrm{II})$ as well as those who did not complete diagnostics by protocol (no expert interview: $n=24$; no indicated second expert interview: $n=13$ ) were excluded. Cohen's Kappa for ADHD versus no ADHD. Cells with false positive/false negative DIVA results are grayed out for reading comfort. DIVA = Diagnostic Interview for ADHD in Adults; Sens. = sensitivity; Spec. = specificity; PPV = positive predictive value; NPV = negative predictive value; DSM-IV = Diagnostic and Statistical Manual of Mental Disorders (4th ed.; APA, I994); DSM-5 = Diagnostic and Statistical Manual of Mental Disorders (5th ed.; APA, 2013); APA = American Psychiatric Association.

$p<.00$ I (chi-square) for all combinations.** highly significant $(p<.0$ I).

2015; Ohlmeier et al., 2008; Reyes et al., 2019; Roncero et al., 2019; van de Glind et al., 2014). A large international multicenter study with 339 inpatients and 351 outpatients with AUD applied a structured interview for ADHD diagnosis (van de Glind et al., 2014) and found an ADHD prevalence rate of $7.5 \%$ ( $6 \%$ inpatients, $9 \%$ outpatients $)$, with a high variability depending on country and setting with inpatient prevalence rates between $5 \%$ and $22 \%$. In a smaller German trial, 91 alcohol dependent inpatients of a psychiatric clinic were included (Ohlmeier et al., 2008). Here, an adult ADHD prevalence rate of $6.6 \%$ was reported by solely applying a self-rating questionnaire to assess adult ADHD symptoms. Johann et al. (2003) used a self-rating questionnaire for childhood and an investigator rated checklist of ADHD symptoms for adulthood on a sample of alcohol dependent inpatients and found adult ADHD to be prevalent in 67 of 314 patients $(21.3 \%)$. However, patients with major psychiatric disorders such as depressive disorders and those with comorbid addictions to drugs other than nicotine were excluded. Reyes et al. (2019) primarily included in- and outpatients with AUD. ADHD diagnosis was assessed by a structured interview and confirmed in 29 of 379 patients (7.7\%). Roncero et al. (2019) conducted a structured interview performed by experienced clinicians on 297 patients with a lifetime diagnosis of alcohol dependence. By extrapolating the results of a screening questionnaire on a larger sample of 729 patients, an ADHD rate of $16.1 \%$ was estimated. One of the highest rates of ADHD (21.1\%) in 355 outpatients with lifetime alcohol dependence was reported in a study (Daigre et al., 2015), which described the most extensive diagnostic work-up of all previous studies. It comprised three successive sessions and included a structured interview on ADHD and another on psychiatric comorbidities, and administration of questionnaires before diagnosis. This high prevalence rate compares well with our study, which also required three successive interviews for ADHD diagnosis and only included patients with prolonged alcohol abstinence. These results may indicate that a more in-depth 
Table 3. Alcohol/lllicit Substance Use Variables for Patients With and Without ADHD Diagnosis (ADHD vs. No ADHD).

\begin{tabular}{|c|c|c|c|c|c|}
\hline & \multicolumn{2}{|c|}{ ADHD $(N=85)$} & \multicolumn{2}{|c|}{ No ADHD $(N=319)$} & \multirow[b]{2}{*}{$p$} \\
\hline & $M / \%(n)$ & $S D$ & $M / \%(n)$ & $S D$ & \\
\hline Age in years on admission & 41.4 & 10.3 & 49.3 & 10.2 & $<.001 * *^{\mathrm{a}}$ \\
\hline Gender in \% male $(n)$ & $76.5 \%(65)$ & & $70.8 \%(226)$ & & $.305^{\mathrm{b}}$ \\
\hline Duration of alcohol dependence in years & 13.1 & 9.0 & 13.5 & 9.0 & $.68 \mathrm{I}^{\mathrm{a}}$ \\
\hline Age of onset of problematic alcohol use & 25.3 & 9.3 & 31.9 & 12.1 & $<.001 * * a$ \\
\hline Age of onset of alcohol use disorder & 26.9 & 10.5 & 34.3 & 11.9 & $<.001 * * a$ \\
\hline History of delirium tremens in \% (n) & $14.1 \%(12)$ & & $6.0 \%(19)$ & & $.012^{* \mathrm{~b}}$ \\
\hline History of withdrawal seizures in \% (n) & $15.3 \%(13)$ & & $13.2 \%(42)$ & & $.611^{\mathrm{b}}$ \\
\hline Severity of alcohol dependence (ADS score) & 18.6 & 6.9 & 12.7 & 6.5 & $<.001 * * c$ \\
\hline AUDIT score & 28.5 & 7.0 & 25.0 & 7.6 & $<.001 * * c$ \\
\hline Drinking days (last 30 days before treatment initiation) & 10.0 & 10.5 & 8.5 & 10.2 & $.219^{\mathrm{a}}$ \\
\hline Tobacco use disorder in \% (n) & $80.0 \%(68)$ & & $69.3 \%(221)$ & & $.052^{b}$ \\
\hline Age of onset of tobacco use disorder & 16.2 & 2.9 & 18.1 & 5.1 & $.010^{* * a}$ \\
\hline Abstinence on admission in \% yes & $88.2 \%(75)$ & & $88.4 \%(282)$ & & $.966^{\mathrm{b}}$ \\
\hline Substance relapse during treatment in \% (n) & $10.6 \%(9)$ & & $4.4 \%(14)$ & & $.036^{* d}$ \\
\hline Age of onset of any substance use disorder (including alcohol and nicotine) & 19.5 & 9.1 & 23.3 & 11.6 & $.001^{\mathrm{a}}$ \\
\hline $\begin{array}{l}\text { Family history of substance use disorder in \% ( } n \text { ) (parents, grandparents, } \\
\text { siblings) }\end{array}$ & $58.8 \%(50)$ & & $42.0 \%(134)$ & & $.006 * * \mathrm{~b}$ \\
\hline Age of onset of drug use disorder (excluding alcohol and nicotine) & 21.0 & 10.4 & 27.3 & 15.3 & $.252^{\mathrm{a}}$ \\
\hline Intravenous consumption ever in \% (n) & $7.1 \%(6)$ & & $1.6 \%(5)$ & & $.014^{*}$ \\
\hline Drug use disorder (except alcohol and nicotine) in \% (n) & $32.9 \%(28)$ & & $11.6 \%(37)$ & & $<.001 * * \mathrm{~b}$ \\
\hline Amphetamine use disorder in \% (n) & $4.7 \%(4)$ & & $0.3 \%(I)$ & & $.008 * * d$ \\
\hline Cannabis use disorder in \% (n) & $23.5 \%(20)$ & & $7.8 \%(25)$ & & $<.001 * * \mathrm{~d}$ \\
\hline Sedative use disorder in \% (n) & $1.2 \%(I)$ & & $2.5 \%(8)$ & & $.691^{\mathrm{b}}$ \\
\hline Opioid use disorder in \% (n) & $1.2 \%(1)$ & & $1.6 \%(5)$ & & $1.0^{\mathrm{d}}$ \\
\hline Cocaine use disorder in $\%(n)$ & $1.2 \%(1)$ & & $0.3 \%(1)$ & & $.377^{d}$ \\
\hline Polydrug use disorder in \% (n) & $8.2 \%(7)$ & & $1.6 \%(5)$ & & $.005^{* * \mathrm{~d}}$ \\
\hline
\end{tabular}

Note. Patients with diagnosis of "suspected ADHD" $(n=I I)$ were excluded from analysis; use disorder = dependence or harmful use (ICD-I0 FIX.2 or FIx.I); ADS = Alcohol Dependence Scale; AUDIT = Alcohol Use Disorder Identification Test; ICD-I0 = International Statistical Classification of Diseases and Related Health Problems-10.

aMann-Whitney U.

${ }^{b}$ Chi-square.

${ }^{\mathrm{c}}$ The $t$ test.

'Fisher's exact test.

*Significant $(\mathrm{p}<.05)$. **Highly significant $(\mathrm{p}<.0 \mathrm{I})$.

investigation for ADHD in abstinent AUD patients leads to a higher rate of ADHD, which would be missed otherwise.

In conclusion, heterogeneous inclusion and exclusion criteria and diagnostic procedures, different sample sizes and settings may have contributed to the high variability of reported ADHD prevalence in AUD patients in recently published studies.

The feasibility of diagnosing ADHD in patients still actively using substances was assessed by only one study. The authors could confirm $>95 \%$ of the diagnoses after achieving abstinence (van Emmerik-van Oortmerssen et al., 2017). Nevertheless, it is of concern that substance use or (prolonged) withdrawal symptoms might inflate ADHD symptomatology in SUD patients (Levin \& Upadhyaya, 2007; Ramos-Quiroga et al., 2019; Sullivan \& RudnikLevin, 2001). Previous studies on ADHD prevalence in
AUD either did not report on abstinence duration (Daigre et al., 2015; Johann et al., 2003) or required only short intervals of alcohol abstinence (Ohlmeier et al., 2008; Reyes et al., 2019) prior to ADHD assessment. Other studies only required intoxication and withdrawal symptoms to be absent at the time of ADHD assessment (Roncero et al., 2019; van de Glind et al., 2014). In contrast, the majority of patients in the present study were abstinent on admission. Together with a very low relapse rate that usually did not lead to treatment discontinuation, it is quite certain that substance use or withdrawal symptoms did not interfere with ADHD diagnosis, in particular as relapse was usually short in duration and diagnostic interviews were postponed under such circumstances. Furthermore, ADHD assessment was not completed with one single interview, but was a continuous process over several weeks and included a vast amount 
of additional information (Table 1). Finally, the diagnostic process started only after 4 weeks of treatment, allowing the patients to extend their duration of abstinence, settle in the new hospital environment, and stabilize from the domestic psychosocial distress. This entire procedure allowed us to exclude symptoms of SUDs and other psychiatric comorbidities as reasons for ADHD symptoms. Hence, ADHD diagnosis in this study appears to be highly reliable.

Finally, most of the ADHD prevalence studies did not report on dropout rates (Daigre et al., 2015; Ohlmeier et al., 2008; Reyes et al., 2019; Roncero et al., 2019). This is of concern, as SUD patients with ADHD are at increased risk of treatment discontinuation and hence study dropout (Levin et al., 2004), which may lead to underestimation of ADHD prevalence, in particular if dropout rates are high. The largest ADHD prevalence study of SUD patients to date (van de Glind et al., 2014; van de Glind et al., 2013) reported a dropout rate of $48.9 \%$ in 2,595 patients. Of note, these dropouts exhibited a higher rate of positive scores on an adult ADHD self-rating questionnaire (Adult ADHD Self-Report Scale [ASRS]), which is suggestive of a higher ADHD prevalence rate in this subgroup. Although the diagnostic process in our study lasted several weeks, we noted low dropout rates and were thus able to fully assess a large proportion $(85.7 \%)$ of all included patients.

Due to our high diagnostic completion rate, only a few patients were not fully assessed. Despite the small number of dropouts, we have found significant differences in some variables for those patients who dropped out of the study after DIVA. The patterns resemble those found in patients with ADHD versus patients without ADHD to some extent, which is not surprising, given the probably higher rate of ADHD in these patients with suspected ADHD diagnosis according to DIVA. Hence, ADHD prevalence rates could have been even higher if those patients had been fully assessed.

By only assessing a subsample of the primary population, a selection bias can lead to wrong prevalence estimations (Delgado-Rodríguez \& Llorca, 2004). Our high study inclusion rate of $66.5 \%$ of all admitted alcohol dependent patients indicates a low risk for such selection bias and underlines the validity of our results.

\section{Substance Use in Patients With ADHD}

Patients with ADHD start their problematic substance use at a significantly younger age (Biederman et al., 2006; Chang, Lichtenstein, \& Larsson, 2012; Young et al., 2015). They are younger when entering treatment but have the same duration of AD as patients without ADHD (Barkley, Fischer, Smallish, \& Fletcher, 2004; King, Brooner, Kidorf, Stoller, \& Mirsky, 1999; Kousha, Shahrivar, \& Alaghband-Rad, 2012). Patients with ADHD are more severely affected by $\mathrm{AD}$, have a higher rate of comorbid SUD (Huntley et al.,
2012; Kousha et al., 2012), and show an increased relapse rate (Ercan et al., 2003; Wilens, Biederman, \& Mick, 1998). All these findings were replicated in our study, which indicates that our study population of residential rehabilitation patients is comparable with other samples of treatment seeking AUD patients.

Children and adolescents with ADHD are at increased risk for smoking and tobacco use disorder (Chang et al., 2012), and tobacco use increases the risk for other SUDs, which is even more pronounced in patients with ADHD (Biederman et al., 2006). In our study, patients with ADHD had a significantly earlier onset of tobacco use disorder and a higher prevalence of tobacco dependence $(p=.052)$.

In our study, patients with ADHD showed an increased rate of positive family history for SUD. This has been reported before (Yule, Wilens, Martelon, Simon, \& Biederman, 2013) and has led to the hypothesis that genetic risk factors for SUD and ADHD overlap (Yule et al., 2017).

In accordance with previous studies, we delineate a severely impaired subgroup of AD patients with previously undiagnosed ADHD which present with early onset alcohol and other substance use, greater severity of alcohol dependence, higher comorbidity with other SUDs, and a higher relapse rate during treatment.

\section{DIVA in AUD Patients}

Structured interviews are often used in scientific and clinical settings and are usually validated by comparing their results with the results of experienced clinicians using official (e.g., DSM-5) criteria. This study is the first in $\mathrm{AD}$ patients that compares ADHD diagnosis from a structured interview with experts' clinical diagnosis. Of note, clinicians evaluated not all patients with DIVA interviews but only those who fulfilled the self-defined threshold criteria for suspicious ADHD, which are considerably lower than the diagnostic thresholds defined by DSM-IV and DSM-5. Hence, we clinically assessed more patients with suspected ADHD from DIVA $(n=146)$ than if we had followed $D S M-I V(n=80)$ or $D S M-5$ criteria $(n=89-104$, depending on number of childhood criteria required).

Results from DIVA showed moderate agreement with clinical ADHD diagnoses. A previous study using clinical expert's diagnosis as gold standard found a better performance for DIVA, but was conducted on a non-SUD sample (Pettersson, Soderstrom, \& Nilsson, 2018). In our study, applying DSM-5 criteria for adulthood (five or more symptoms) and five childhood symptoms showed superior results compared with the stricter $D S M-I V$ criteria (six or more for both adult- and childhood). Adult ADHD patients often have trouble remembering their childhood symptoms (Miller, Newcorn, \& Halperin, 2010), which might be even more pronounced in patients with SUD. A too strict 
threshold for retrospective childhood symptomatology might therefore lead to an underestimation of ADHD.

Adults with ADHD tend to underreport their current ADHD symptoms (Sibley et al., 2012), as they exhibit low self-perception and self-awareness (Manor et al., 2012), are less able to link ADHD symptoms to impairments (Morstedt, Corbisiero, Bitto, \& Stieglitz, 2015), and often compensate their deficits to some extent, which masks their symptoms and makes it more difficult to diagnose ADHD (Adler \& Cohen, 2004; Culpepper \& Mattingly, 2008; Kalbag \& Levin, 2005). This pattern of underreporting symptoms occurs in children with ADHD as well and has been described as typical for ADHD (Owens, Goldfine, Evangelista, Hoza, \& Kaiser, 2007). Hence, successive diagnostic interviews over the course of several weeks as performed in the present study might have led to improved introspection regarding ADHD symptoms in patients over time, which could partly explain the differences between the initial DIVA results and the subsequent experts' results. Also, many of the additional information sources (e.g., school records, parents' rating scales) were only available to the experts. Several guidelines emphasize the fact that ADHD diagnosis should be based on as many sources as possible (American Academy of Pediatrics, 2011; Kalbag \& Levin, 2005; S. J. Kooij et al., 2010; National Collaborating Centre for Mental Health, 2009), which is supported by our study results.

According to our results, DIVA can be used in $\mathrm{AD}$ patients to assess ADHD symptomatology yet is accompanied by a significant rate of false negative and false positive results necessitating additional assessment by experienced clinicians. As DIVA previously showed a 100\% agreement with another commonly used structured interview (RamosQuiroga et al., 2019), a careful interpretation of the results of structured ADHD interviews in the SUD population appears to be warranted in general.

\section{Conclusion}

The high prevalence of previously undiagnosed ADHD in every fifth alcohol dependent patient in residential alcohol treatment is highly relevant for the diagnostic assessment and therapy. It ought to encourage AUD treatment facilities to implement standard diagnostic procedures for all admitted patients to reliably identify undiagnosed ADHD. As there are both effective psychotherapeutic and medical treatment options available for patients with ADHD with comorbid SUD (Aviram, Rhum, \& Levin, 2001; Grant et al., 2015; van Emmerik-van Oortmerssen, Vedel, van den Brink, \& Schoevers, 2015; Wilens et al., 2008), identification of this large subgroup of severely impaired AD patients will hopefully lead to optimized treatment strategies with improved treatment outcomes for both $\mathrm{AD}$ and $\mathrm{ADHD}$.

\section{Declaration of Conflicting Interests}

The author(s) declared no potential conflicts of interest with respect to the research, authorship, and/or publication of this article.

\section{Funding}

The author(s) received no financial support for the research, authorship, and/or publication of this article.

\section{Supplemental Material}

Supplementary material for this article is available online.

\section{ORCID iD}

M Luderer (iD) http://orcid.org/0000-0002-6364-9136

\section{References}

Adler, L., \& Cohen, J. (2004). Diagnosis and evaluation of adults with attention-deficit/hyperactivity disorder. Psychiatric Clinics of North America, 27, 187-201. doi:10.1016/j. psc.2003.12.003

American Academy of Pediatrics. (2011). ADHD: Clinical practice guideline for the diagnosis, evaluation, and treatment of attention-deficit/hyperactivity disorder in children and adolescents. Pediatrics, 128, 1007-1022. doi:10.1542/peds.2011-2654

American Psychiatric Association. (1994). Diagnostic and statistical manual of mental disorders (4th ed.). Washington, DC: Author.

American Psychiatric Association. (2013). Diagnostic and statistical manual of mental disorders (5th ed.). Arlington, VA: American Psychiatric Publishing.

Arias, A. J., Gelernter, J., Chan, G., Weiss, R. D., Brady, K. T., Farrer, L., \& Kranzler, H. R. (2008). Correlates of cooccurring ADHD in drug-dependent subjects: Prevalence and features of substance dependence and psychiatric disorders. Addictive Behaviors, 33, 1199-1207. doi:10.1016/j.addbeh.2008.05.003

Aviram, R. B., Rhum, M., \& Levin, F. R. (2001). Psychotherapy of adults with comorbid attention-deficit/hyperactivity disorder and psychoactive substance use disorder. Journal of Psychotherapy Practice and Research, 10, 179-186.

Barkley, R. A., Fischer, M., Smallish, L., \& Fletcher, K. (2004). Young adult follow-up of hyperactive children: Antisocial activities and drug use. Journal of Child Psychology and Psychiatry, 45, 195-211.

Biederman, J., Monuteaux, M. C., Mick, E., Wilens, T. E., Fontanella, J. A., Poetzl, K. M., . . Faraone, S. V. (2006). Is cigarette smoking a gateway to alcohol and illicit drug use disorders? A study of youths with and without attention deficit hyperactivity disorder. Biological Psychiatry, 59, 258-264. doi:10.1016/j.biopsych.2005.07.009

Capusan, A. J., Bendtsen, P., Marteinsdottir, I., Kuja-Halkola, R., \& Larsson, H. (2015). Genetic and environmental contributions to the association between attention deficit hyperactivity disorder and alcohol dependence in adulthood: A large population-based twin study. American Journal of Medical Genetics, Part B: Neuropsychiatric Genetics, 168, 414-422. doi:10.1002/ajmg.b.32300 
Carroll, K. M., \& Rounsaville, B. J. (1993). History and significance of childhood attention deficit disorder in treatment-seeking cocaine abusers. Comprehensive Psychiatry, 34, 75-82.

Chang, Z., Lichtenstein, P., \& Larsson, H. (2012). The effects of childhood ADHD symptoms on early-onset substance use: A Swedish twin study. Journal of Abnormal Child Psychology, 40, 425-435. doi:10.1007/s10802-011-9575-6

Conners, C. K., Erhardt, D., \& Sparrow, E. (1999). Conners Adult ADHD Rating Scales (CAARS): Technical manual. New York, NY: Multi Health Systems.

Culpepper, L., \& Mattingly, G. (2008). A practical guide to recognition and diagnosis of ADHD in adults in the primary care setting. Postgraduate Medicine, 120(3), 16-26. doi:10.3810/ pgm.2008.09.1904

Daigre, C., Roncero, C., Rodriguez-Cintas, L., Ortega, L., Lligona, A., Fuentes, S., . . . Casas, M. (2015). Adult ADHD screening in alcohol-dependent patients using the Wender-Utah Rating Scale and the adult ADHD SelfReport Scale. Journal of Attention Disorders, 19, 328-334. doi:10.1177/1087054714529819

Davis, C., Cohen, A., Davids, M., \& Rabindranath, A. (2015). Attention-deficit/hyperactivity disorder in relation to addictive behaviors: A moderated-mediation analysis of personality-risk factors and sex. Frontiers in Psychiatry, 6, 47. doi:10.3389/fpsyt.2015.00047

De Alwis, D., Lynskey, M. T., Reiersen, A. M., \& Agrawal, A. (2014). Attention-deficit/hyperactivity disorder subtypes and substance use and use disorders in NESARC. Addictive Behaviors, 39, 1278-1285. doi:10.1016/j.addbeh.2014.04.003

Delgado-Rodríguez, M., \& Llorca, J. (2004). Bias. Journal of Epidemiology and Community Health, 58, 635-641. doi:10.1136/jech.2003.008466

Edwards, A. C., \& Kendler, K. S. (2012). Twin study of the relationship between adolescent attention-deficit/hyperactivity disorder and adult alcohol dependence. Journal of Studies on Alcohol and Drugs, 73, 185-194.

Ercan, E. S., Coskunol, H., Varan, A., \& Toksoz, K. (2003). Childhood attention deficit/hyperactivity disorder and alcohol dependence: A 1-year follow-up. Alcohol and Alcoholism, 38, 352-356.

Grant, B. F., Goldstein, R. B., Saha, T. D., Chou, S. P., Jung, J., Zhang, H., . . Hasin, D. S. (2015). Epidemiology of DSM-5 alcohol use disorder: Results from the national epidemiologic survey on alcohol and related conditions III. JAMA Psychiatry, 72, 757-766. doi:10.1001/jamapsychiatry.2015.0584

Heilig, M., Egli, M., Crabbe, J. C., \& Becker, H. C. (2010). Acute withdrawal, protracted abstinence and negative affect in alcoholism: Are they linked? Addiction Biology, 15, 169-184. doi:10.1111/j.1369-1600.2009.00194.x

Hirth, N., Meinhardt, M. W., Noori, H. R., Salgado, H., Torres-Ramirez, O., Uhrig, S., . . . Hansson, A. C. (2016). Convergent evidence from alcohol-dependent humans and rats for a hyperdopaminergic state in protracted abstinence. Proceedings of the National Academy of Sciences, 113, 30243029. doi:10.1073/pnas. 1506012113

Huntley, Z., Maltezos, S., Williams, C., Morinan, A., Hammon, A., Ball, D., . . Asherson, P. J. (2012). Rates of undiagnosed attention deficit hyperactivity disorder in London drug and alcohol detoxification units. BMC Psychiatry, 12, Article 223. doi:10.1186/1471-244X-12-223

Johann, M., Bobbe, G., Putzhammer, A., \& Wodarz, N. (2003). Comorbidity of alcohol dependence with attention-deficit hyperactivity disorder: Differences in phenotype with increased severity of the substance disorder, but not in genotype (serotonin transporter and 5-hydroxytryptamine-2c receptor). Alcoholism: Clinical and Experimental Research, 27, 15271534. doi:10.1097/01.ALC.0000090143.00703.07

Kalbag, A. S., \& Levin, F. R. (2005). Adult ADHD and substance abuse: Diagnostic and treatment issues. Substance Use \& Misuse, 40, 1955-1981, 2043-2048. doi:10.1080/ 10826080500294858

Kessler, R. C., Adler, L., Ames, M., Demler, O., Faraone, S., Hiripi, E., . . . Spencer, T. (2005). The World Health Organization Adult ADHD Self-Report Scale (ASRS): A short screening scale for use in the general population. Psychological Medicine, 35, 245-256.

King, V. L., Brooner, R. K., Kidorf, M. S., Stoller, K. B., \& Mirsky, A. F. (1999). Attention deficit hyperactivity disorder and treatment outcome in opioid abusers entering treatment. Journal of Nervous and Mental Disease, 187, 487-495.

Kooij, J. J. S. (2012). Diagnostic Interview for ADHD in Adults 2.0 (DIVA 2.0). In J.J. Sandra Kooij (ed.) Adult ADHD: Diagnostic Assessment and Treatment. London, England: Springer.pp. 98-99.

Kooij, S. J., Bejerot, S., Blackwell, A., Caci, H., Casas-Brugue, M., Carpentier, P. J., . . . Asherson, P. (2010). European consensus statement on diagnosis and treatment of adult ADHD: The European Network Adult ADHD. BMC Psychiatry, 10, Article 67. doi:10.1186/1471-244X-10-67

Kousha, M., Shahrivar, Z., \& Alaghband-Rad, J. (2012). Substance use disorder and ADHD: Is ADHD a particularly "specific" risk factor? Journal of Attention Disorders, 16, 325-332. doi: $10.1177 / 1087054710387265$

Krause, K.-H., Krause, J., \& Trott, G.-E. (1998). [Hyperkinetic syndrome (attention deficit-/hyperactivity disorder) in adulthood]. Der Nervenarzt, 69(7), 543-556. doi:10.1007/ $\mathrm{s} 001150050311$

Lee, S. S., Humphreys, K. L., Flory, K., Liu, R., \& Glass, K. (2011). Prospective association of childhood attention-deficit/ hyperactivity disorder (ADHD) and substance use and abuse/ dependence: A meta-analytic review. Clinical Psychology Review, 31, 328-341. doi:10.1016/j.cpr.2011.01.006

Levin, F. R., Evans, S. M., Vosburg, S. K., Horton, T., Brooks, D., \& Ng, J. (2004). Impact of attention-deficit hyperactivity disorder and other psychopathology on treatment retention among cocaine abusers in a therapeutic community. Addictive Behaviors, 29, 1875-1882. doi:10.1016/j.addbeh.2004.03.041

Levin, F. R., \& Upadhyaya, H. P. (2007). Diagnosing ADHD in adults with substance use disorder: DSM-IV criteria and differential diagnosis. Journal of Clinical Psychiatry, 68(7), e18.

Manor, I., Vurembrandt, N., Rozen, S., Gevah, D., Weizman, A., \& Zalsman, G. (2012). Low self-awareness of ADHD in adults using a self-report screening questionnaire. European Psychiatry, 27, 314-320. doi:10.1016/j.eurpsy.2010.08.013

McAweeney, M., Rogers, N. L., Huddleston, C., Moore, D., \& Gentile, J. P. (2010). Symptom prevalence of ADHD in a community residential substance abuse treatment 
program. Journal of Attention Disorders, 13, 601-608. doi:10.1177/1087054708329973

McHugh, M. L. (2012). Interrater reliability: The kappa statistic. Biochemia Medica, 22, 276-282.

Miller, C. J., Newcorn, J. H., \& Halperin, J. M. (2010). Fading memories: Retrospective recall inaccuracies in ADHD. Journal of Attention Disorders, 14, 7-14. doi:10.1177/ 1087054709347189

Morstedt, B., Corbisiero, S., Bitto, H., \& Stieglitz, R. D. (2015). Attention-deficit/hyperactivity disorder (ADHD) in adulthood: Concordance and differences between self- and informant perspectives on symptoms and functional impairment. PLoS ONE, 10(11), e0141342. doi:10.1371/journal. pone. 0141342

Moura, H. F., Faller, S., Benzano, D., Szobot, C., von Diemen, L., Stolf, A. R., . . Kessler, F. H. (2013). The effects of ADHD in adult substance abusers. Journal of Addictive Diseases, 32, 252-262. doi:10.1080/10550887.2013.824359

National Collaborating Centre for Mental Health. (2009). Attention deficit hyperactivity disorder: The NICE guideline on diagnosis and management of ADHD in children, young people and adults. London, England.

Ohlmeier, M. D., Peters, K., Te Wildt, B. T., Zedler, M., Ziegenbein, M., Wiese, B., . . .Schneider, U. (2008). Comorbidity of alcohol and substance dependence with attention-deficit/hyperactivity disorder (ADHD). Alcohol and Alcoholism, 43, 300-304. doi:10.1093/alcalc/agn014

Owens, J. S., Goldfine, M. E., Evangelista, N. M., Hoza, B., \& Kaiser, N. M. (2007). A critical review of self-perceptions and the positive illusory bias in children with ADHD. Clinical Child and Family Psychology Review, 10, 335-351. doi:10.1007/s10567-007-0027-3

Pedersen, S. L., Walther, C. A., Harty, S. C., Gnagy, E. M., Pelham, W. E., \& Molina, B. S. (2016). The indirect effects of childhood attention deficit hyperactivity disorder on alcohol problems in adulthood through unique facets of impulsivity. Addiction, 111, 1582-1589. doi:10.1111/add.13398

Pettersson, R., Soderstrom, S., \& Nilsson, K. W. (2018). Diagnosing ADHD in adults: An examination of the discriminative validity of neuropsychological tests and diagnostic assessment instruments. Journal of Attention Disorders, 22 , 1019-1031. doi:10.1177/1087054715618788

Polanczyk, G. V., Salum, G. A., Sugaya, L. S., Caye, A., \& Rohde, L. A. (2015). Annual research review: A meta-analysis of the worldwide prevalence of mental disorders in children and adolescents. Journal of Child Psychology and Psychiatry, 56, 345-365. doi:10.1111/jcpp.12381

Ramos-Quiroga, J. A., Nasillo, V., Richarte, V., Corrales, M., Palma, F., Ibanez, P., . . Kooij, J. J. (2019). Criteria and concurrent validity of DIVA 2.0: A semi-structured diagnostic interview for adult ADHD. Journal of Attention Disorders, 23, 1126-1135. doi:10.1177/1087054716646451

Retz-Junginger, P., Retz, W., Blocher, D., Stieglitz, R. D., Georg, T., Supprian, T., ... Rosler, M. (2003). [Reliability and validity of the Wender-Utah-Rating-Scale short form. Retrospective assessment of symptoms for attention deficit/hyperactivity disorder]. Der Nervenarzt, 74, 987-993. doi:10.1007/s00115$002-1447-4$
Reyes, M. M., Schneekloth, T. D., Hitschfeld, M. J., Geske, J. R., Atkinson, D. L., \& Karpyak, V. M. (2019). The clinical utility of ASRS-v1.1 for identifying ADHD in alcoholics using PRISM as the reference standard. Journal of Attention Disorders, 23, 1119-1125. doi:10.1177/1087054716646450

Roberts, W., Peters, J. R., Adams, Z. W., Lynam, D. R., \& Milich, R. (2014). Identifying the facets of impulsivity that explain the relation between ADHD symptoms and substance use in a nonclinical sample. Addictive Behaviors, 39, 1272-1277. doi:10.1016/j.addbeh.2014.04.005

Roncero, C., Ortega, L., Perez-Pazos, J., Lligona, A., Abad, A. C., Gual, A., . . . Daigre, C. (2019). Psychiatric comorbidity in treatment-seeking alcohol dependence patients with and without ADHD. Journal of Attention Disorders, 23, 1497-1504. doi:10.1177/1087054715598841

Rosler, M., Retz, W., Retz-Junginger, P., Thome, J., Supprian, T., Nissen, T., . . . Trott, G. E. (2004). [Tools for the diagnosis of attention-deficit/hyperactivity disorder in adults. Selfrating behaviour questionnaire and diagnostic checklist]. Der Nervenarzt, 75, 888-895. doi:10.1007/s00115-003-1622-2

Saunders, J. B., Aasland, O. G., Babor, T. F., de la Fuente, J. R., \& Grant, M. (1993). Development of the Alcohol Use Disorders Identification Test (AUDIT): WHO collaborative project on early detection of persons with harmful alcohol consumptionII. Addiction, 88, 791-804.

Sibley, M. H., Pelham, W. E., Molina, B. S., Gnagy, E. M., Waxmonsky, J. G., Waschbusch, D. A., . . Kuriyan, A. B. (2012). When diagnosing ADHD in young adults emphasize informant reports, DSM items, and impairment. Journal of Consulting and Clinical Psychology, 80, 1052-1061. doi:10.1037/a0029098

Simon, V., Czobor, P., Balint, S., Meszaros, A., \& Bitter, I. (2009). Prevalence and correlates of adult attention-deficit hyperactivity disorder: Meta-analysis. British Journal of Psychiatry, 194, 204-211. doi:10.1192/bjp.bp.107.048827

Skinner, H. A., \& Horn, J. L. (1984). Alcohol Dependence Scale (ADS) user's guide. Toronto, Ontario, Canada: Addiction Research Foundation.

Spanagel, R., Noori, H. R., \& Heilig, M. (2014). Stress and alcohol interactions: Animal studies and clinical significance. Trends in Neurosciences, 37, 219-227. doi:10.1016/j. tins.2014.02.006

Sullivan, M. A., \& Rudnik-Levin, F. (2001). Attention deficit/hyperactivity disorder and substance abuse. Diagnostic and therapeutic considerations. ANNALS of the New York Academy of Sciences, 931, 251-270.

van de Glind, G., Konstenius, M., Koeter, M. W., van Emmerikvan Oortmerssen, K., Carpentier, P. J., Kaye, S., . . . Group, I. R. (2014). Variability in the prevalence of adult ADHD in treatment seeking substance use disorder patients: Results from an international multi-center study exploring DSM-IV and DSM-5 criteria. Drug and Alcohol Dependence, 134, 158-166. doi:10.1016/j.drugalcdep.2013.09.026

van de Glind, G., Van Emmerik-van Oortmerssen, K., Carpentier, P. J., Levin, F. R., Koeter, M. W., Barta, C., . . . van den Brink, W. (2013). The International ADHD in Substance Use Disorders Prevalence (IASP) study: Background, methods and study population. International 
Journal of Methods in Psychiatric Research, 22, 232-244. doi:10.1002/mpr.1397

van Emmerik-van Oortmerssen, K., van de Glind, G., van den Brink, W., Smit, F., Crunelle, C. L., Swets, M., \& Schoevers, R. A. (2012). Prevalence of attention-deficit hyperactivity disorder in substance use disorder patients: A meta-analysis and meta-regression analysis. Drug and Alcohol Dependence, 122, 11-19. doi:10.1016/j.drugalcdep.2011.12.007

van Emmerik-van Oortmerssen, K., Vedel, E., Kramer, F. J., Koeter, M. W., Schoevers, R. A., \& van den Brink, W. (2017). Diagnosing ADHD during active substance use: Feasible or flawed? Drug and Alcohol Dependence, 180, 371-375. doi:10.1016/j.drugalcdep.2017.07.039

van Emmerik-van Oortmerssen, K., Vedel, E., van den Brink, W., \& Schoevers, R. A. (2015). Integrated cognitive behavioral therapy for patients with substance use disorder and comorbid ADHD: Two case presentations. Addictive Behaviors, 45, 214-217. doi:10.1016/j.addbeh.2015.01.040

Vogel, T., Dom, G., van de Glind, G., Studer, J., Gmel, G., Strik, W., \& Moggi, F. (2016). Is attention deficit/hyperactivity disorder among men associated with initiation or escalation of substance use at 15-month follow-up? A longitudinal study involving young Swiss men. Addiction, 111, 1867-1878. doi:10.1111/add.13422

Ward, M. F., Wender, P. H., \& Reimherr, F. W. (1993). The Wender Utah Rating Scale: An aid in the retrospective diagnosis of childhood attention deficit hyperactivity disorder. American Journal of Psychiatry, 150, 885-890. doi:10.1176/ajp.150.6.885

Wender, P. H. (1995). Attention-deficit hyperactivity disorder in adults. Oxford, UK: Oxford University Press.

Wickens, J. R., \& Tripp, E. G. (2005). Altered sensitivity to reward in children with ADHD: Dopamine timing is off. Behavioral and Brain Sciences, 28, 445-446.

Wilens, T. E., Adler, L. A., Weiss, M. D., Michelson, D., Ramsey, J. L., Moore, R. J., . . . Levine, L. R. (2008). Atomoxetine treatment of adults with ADHD and comorbid alcohol use disorders. Drug and Alcohol Dependence, 96, 145-154. doi:10.1016/j.drugalcdep.2008.02.009

Wilens, T. E., Biederman, J., \& Mick, E. (1998). Does ADHD affect the course of substance abuse? Findings from a sample of adults with and without ADHD. American Journal on Addictions, 7, 156-163.

Wilens, T. E., \& Morrison, N. R. (2011). The intersection of attention-deficit/hyperactivity disorder and substance abuse. Current Opinion in Psychiatry, 24, 280-285. doi:10.1097/ YCO.0b013e328345c956

World Health Organization. (1993). The ICD-10 classification of mental and behavioural disorders: Diagnostic criteria for research. Geneva, Switzerland: Author.
Young, J. T., Carruthers, S., Kaye, S., Allsop, S., Gilsenan, J., Degenhardt, L., . . . Preen, D. (2015). Comorbid attention deficit hyperactivity disorder and substance use disorder complexity and chronicity in treatment-seeking adults. Drug and Alcohol Review, 34, 683-693. doi:10.1111/dar.12249

Yule, A. M., Martelon, M., Faraone, S. V., Carrellas, N., Wilens, T. E., \& Biederman, J. (2017). Examining the association between attention deficit hyperactivity disorder and substance use disorders: A familial risk analysis. Journal of Psychiatric Research, 85, 49-55. doi:10.1016/j.jpsychires.2016.10.018

Yule, A. M., Wilens, T. E., Martelon, M. K., Simon, A., \& Biederman, J. (2013). Does exposure to parental substance use disorders increase substance use disorder risk in offspring? A 5-year follow-up study. American Journal on Addictions, 22, 460-465. doi:10.1111/j.1521-0391.2013.12048.x

\section{Author Biographies}

Mathias Luderer received his medical degree from Heidelberg University, Germany, and is a board approved specialist in psychiatry and psychotherapy. He is treating and investigating the comorbidity of ADHD in patients with substance use disorders since 2010 at the Central Institute of Mental Health (CIMH), Department of Addictive Behavior and Addiction Medicine, Mannheim, Germany.

Christian Sick, MBA, graduated at Albert Ludwig University Freiburg, Germany, in medicine and Bayreuth University, Germany, in applied economics. He is a board approved neurologist and health economist. He is a doctoral candidate.

Nurcihan Kaplan-Wickel received her medical degree from Heidelberg University, Germany, and is a doctoral candidate.

Iris Reinhard graduated in mathematics at Heidelberg University and has many years of statistical experience in psychiatric and addiction research at the CIMH Biostatistics Department.

Agnes Richter is a research assistant at MEDIAN Klinik Wilhelmsheim, Germany.

Falk Kiefer is a professor of psychiatry and psychotherapy, chair of addiction research, and director of the Department of Addictive Behavior and Addiction Medicine at the CIMH. He has over 20 years of experience in clinical and neurobiological addiction research

Tillmann Weber is a board approved specialist in psychiatry and psychotherapy and head of the addiction treatment center MEDIAN Klinik Wilhelmsheim, Germany, where the study was conducted. He has over 15 years of experience in basic and clinical neuropsychiatric and addiction research. 